\title{
Prominence Mapping of the RS CVn system HR 1099
}

\author{
P. Petit ${ }^{1}$, J.-F Donati ${ }^{2}$, M. Jardine ${ }^{3}$ and A. Collier Cameron ${ }^{3}$ \\ ${ }^{1}$ Max-Planck-Institut für Sonnensystemforschung, Max-Planck-Str. 2, \\ 37191 Katlenburg-Lindau, Germany, email: petit@linmpi.mpg.de \\ ${ }^{2}$ Observatoire Midi-Pyrénées, 14 avenue Edouard Belin, 31400 Toulouse, France, email: \\ donati@ast.obs-mip.fr \\ ${ }^{3}$ School of Physics and Astronomy, University of Saint Andrews, Saint Andrews KY16 9SS, \\ UK, email: mmj@st-and.ac.uk, acc4@st-and.ac.uk
}

\begin{abstract}
We investigate temporal fluctuations in the $\mathrm{H} \alpha$ emission profiles of the RS CVn system HR 1099 from a monitoring using the MuSiCoS spectropolarimeter (Observatoire du Pic du Midi, France) in 2001, between December 01 and December 18. Part of the observed emission fluctuations is consistent with rotational modulation, which we interpret as the spectral signature of a dense and complex prominence system trapped in the magnetosphere of HR 1099 and forced to co-rotate with the binary system. The distribution of emitting material is mapped by means of Doppler tomography. We discuss the evolution of prominences over the observing window.
\end{abstract}

Keywords. stars: flare, stars: activity

\section{Observations}

A total of 164 high-resolution spectra of the RS CVn system HR 1099 was collected in 2001, between Dec. 01 and Dec. 18, with the MuSiCoS spectropolarimeter (Donati et al. 1999) at Observatoire du Pic du Midi (France). The $\mathrm{H} \alpha$ line of the system is always in emission with a peaked, asymmetric, fast-changing profile revealing frequent flaring events (Foing et al. 1996). On sufficiently small timescales, and as long as the system is observed during a quiescent state, most of the structures seen in $\mathrm{H} \alpha$ repeat over several rotation cycles. It is therefore possible to reconstruct the spatial distribution of the emitting material by Doppler tomography. We use here the maximum entropy code developed by Marsh \& Horne (1988) and Donati et al. (2000).

\section{Doppler tomography of $\mathbf{H} \alpha$ emission}

Fig. 1 shows the distribution of $\mathrm{H} \alpha$ emitting material in the velocity space. Two closeby epochs are considered, each one providing a good rotational coverage of the system. In the case of co-rotating structures, velocity maps represent a straightforward image of spatial distribution. Most of the co-rotating clouds are reconstructed around the extremely active primary sub-giant. Prominences are mostly concentrated as a single high-contrast structure in the first epoch, while their distribution is more diffuse in the second map.

A comparison between observed and synthetic line profiles shows that a large part of the profile fluctuations are correctly reproduced by the imaging procedure. Fast, nonrotational changes in the profile shape (as observed in particular at epoch 2) have only a marginal impact on the final map. 
Prominences (Dec. $01--$ Dec. 09)

Prominences (Dec. $10--$ Dec. 18)
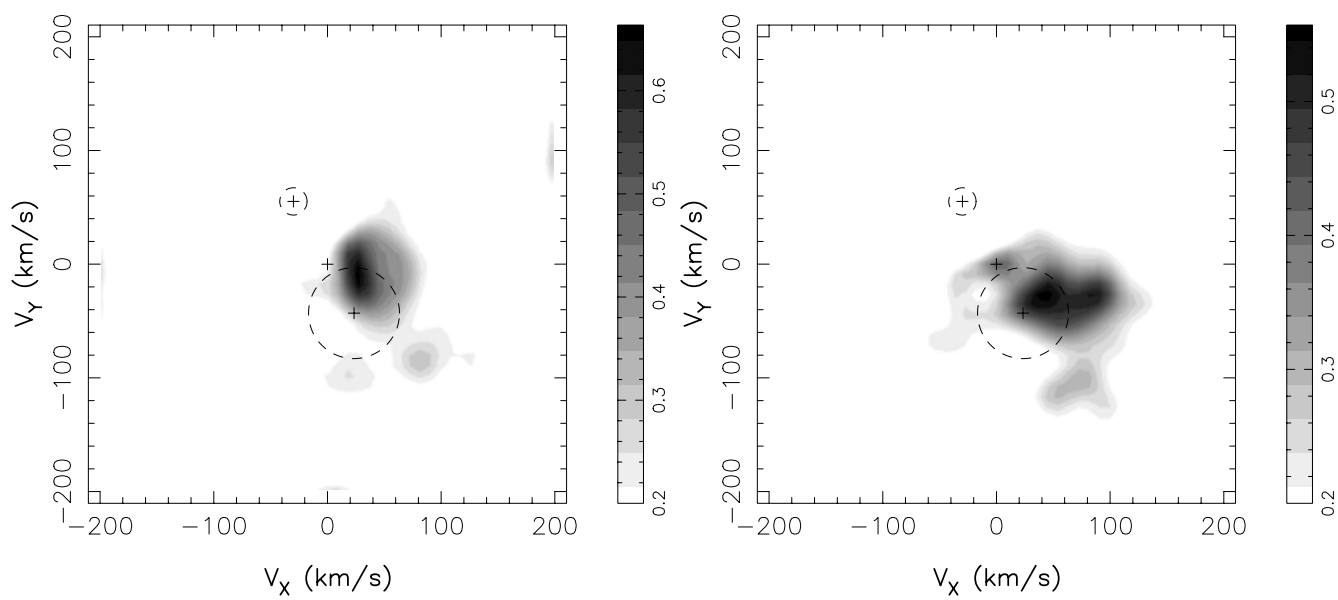

Figure 1. Doppler maps of the $\mathrm{H} \alpha$-emitting material of HR 1099 in the velocity space, as reconstructed from data sets collected between Dec. 01 and Dec. 09 (left) and between Dec. 10 and Dec. 18 (right). The photosphere of both components of the binary system are represented by dashed circles of radius v.sini. The K1 primary subgiant shows up on bottom-right. The G5 secondary dwarf is on top-left. The center of mass of the system is shown as a cross at null velocity.

\section{Future work}

The spatial distribution of prominences provides valuable information about the magnetic geometry of the corona of HR 1099. A photospheric magnetic map of the primary star was reconstructed from the same data set as that employed for $\mathrm{H} \alpha$ tomography (Petit et al.2004) and can be used as boundary conditions to extrapolate the three-dimensional structure of the coronal field (Jardine et al. 2002). Simple assumptions on the field geometry of the secondary component can also be derived from the measurements of Donati (1999). The resulting coronal topology will then be tested by comparison with prominence maps.

Nearly simultaneous observations of HR 1099 obtained at the Anglo-Australian Telescope are available and should allow us to enlarge our observing window up to early January of 2002. Preliminary work demonstrates that intrinsic variability is much more important in this data set, to a point where the basic assumption of co-rotation of circumstellar material does not hold, even over short timescales. Further work is needed to extract an adequate sub-set and possibly merge it with MuSiCoS spectra.

\section{References}

Donati, J.-F. 1999, MNRAS 302, p. 457

Donati, J.-F., Mengel, M., Carter, B.D., Marsden, S., Collier Cameron, A., \& Wichmann, R. 2000, MNRAS 316, p. 699

Foing, B. H., et al. 1996, IAU Colloq. 153: Magnetodynamic Phenomena in the Solar Atmosphere - Prototypes of Stellar Magnetic Activity, p. 283

Jardine, M., Collier Cameron, A., \& Donati, J.-F. 2002, MNRAS 333, p. 339

Marsh, T. R. \& Horne, K. 1988, MNRAS 235, p. 269

Petit, P., et al. 2004, MNRAS 348, p. 1175 